\title{
Rencontre avec Jorge Lavelli, metteur en scène de Macbeth, d'E. Ionesco
}

\section{(2) OpenEdition \\ 12 Journals}

\section{Édition électronique}

URL : http://journals.openedition.org/shakespeare/1284

DOI : $10.4000 /$ shakespeare.1284

ISSN : 2271-6424

Éditeur

Société Française Shakespeare

\section{Édition imprimée}

Date de publication : 1 novembre 1994

Pagination : 225-234

\section{Référence électronique}

"Rencontre avec Jorge Lavelli, metteur en scène de Macbeth, d'E. Ionesco », Actes des congrès de la Société française Shakespeare [En ligne], 12 | 1994, mis en ligne le 01 janvier 2007, consulté le 05 mai 2019. URL : http://journals.openedition.org/shakespeare/1284; DOI : 10.4000/shakespeare.1284 
COSMOPOLITISME ET INSULARITÉ 


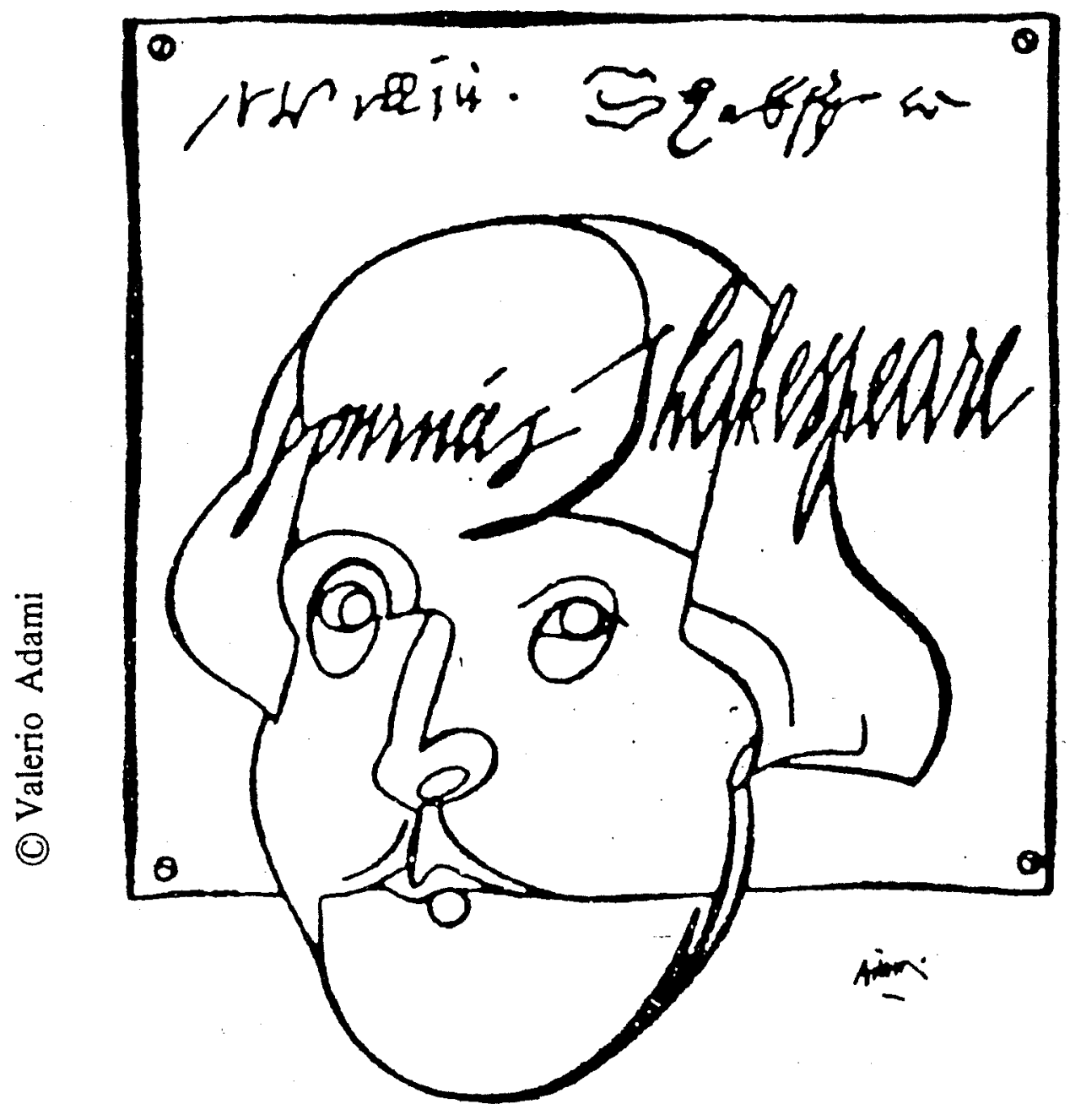

Affiche de Valerio Adami, spécialement conçue pour les journées Shakespeare 1979 


\section{SHAKESPEARE}

COSMOPOLITISME ET INSULARITÉ

Société Française Shakespeare

Actes du Congrès 1993

sous la direction

de

M. T. JONES-DAVIES

Ouvrage publié avec le soutien de

BARCLAYS

BARCLAYS BANK S.A

PARIS

LES BELLES LETTRES

1994 
Tous droits de traduction, de reproduction et d'adaptation réservés pour tous les pays.

(C) 1994 Société d'édition Les Belles Lettres, 95 bd Raspail 75006 Paris

ISBN 2.251.69122-7 


\title{
Rencontre avec Jorge Lavelli, metteur en scène de Macbett d'E. Ionesco
}

\author{
animée par Jean-Michel Déprats
}

JMD. : Madame Jones-Davies m'a demandé de présenter brièvement J. Lavelli et d'animer la rencontre.

Vous avez certainement vu de nombreuses mises en scène de pièce ou d'opéras de J. Lavelli. Le plus grand titre de gloire de J. Lavelli actuellement est d'avoir tenu le pari de diriger un théâtre qui fonctionne uniquement sur un répertoire contemporain et qui a fidélisé des centaines de spectateurs, "le Théâtre de la Colline".

Le trimestre dernier, J. Lavelli a monté Macbett d'Ionesco. La première question serait : comment définirait-il le rapport qu'entretient cette pièce avec le modèle shakespearien ?

$\mathrm{JL}$ : D'abord il faut dire que toutes les présentations du théâtre contemporain ne nous aident pas à parler de Shakespeare. Shakespeare n'est pas présent dans mon "Théâtre de la Colline", en revanche un auteur comme Ionesco prend de droit sa propre place dans la dramaturgie d'après-guerre, il a apporté une immense ouverture en introduisant une dramaturgie qui va à l'encontre de tout psychologisme, de toute construction bourgeoise du théâtre. Il apporte, non seulement au niveau de l'écriture, mais surtout au niveau de l'imaginaire, des thèmes qu'on n'avait pas ou peu vus sur une scène dramatique. Ionesco apporte l'obsession au théâtre, et aussi un jeu avec les mots qui souvent devient significatif ou signifiant de par sa seule utilisation et passe à travers l'explication pour nous mettre devant des faits ou des situations dramatiques accomplis. Beaucoup de ses thèmes sont réguliers, constants : cette obsession de la mort évidemment; la métamorphose aussi, qui, depuis ses premières pièces jusqu'à la fin de ses productions, a été un de ses thèmes-clé. Ce qui donne à l'auteur, par le truchement de ses thèmes désirés, l'emprise sur l'identité.

Si Ionesco s'est intéressé à Shakespeare, et surtout à cette pièce, Macbeth, c est parcequ'il y voyait des points communs : les sorcières shakespeariennes et les sorcières d'Ionesco ont un côté in- 
cantatoire, qui était loin d'être contraire a sa manière de considérer le thêâtre. Globalement, en introduisant non seulement une sorte de dérision, mais aussi en grossissant des traits, Ionesco arrive à emprunter totalement Shakespeare, mais pour faire une œuvre personnelle. Cela échappe à ce que l'on peut appeler une adaptation ou un exercice de style. Voilà ce qui est le plus intéressant dans cette production. C'est cent pour cent Ionesco, tout en restant quelque part très proche de Shakespeare, - l'écriture est tout à fait lonesco, la pensée aussi, la simplification, les grossissements, l'outrance, le sens de la dérision. Il reste une œuvre intéressante parce qu'Ionesco est un auteur dont la dramaturgie est basée sur l'immobilité. Il est très difficile parfois de raconter une pièce d'Ionesco : il se passe beaucoup de choses, mais en fait il ne se passe rien du point de vue anecdotique. Comment peut-on raconter Les Chaises? il y a de nombreux événements, mais en même temps on est au niveau de l'inconscient, donc on ne peut pas raconter comme on raconte une œuvre d'un auteur psychologique ou bien une œuvre de Shakespeare. Le fait de prendre Shakespeare l'a obligé à suivre une certaine anecdote, la pièce d'Ionesco en devient plus attrayante, car c'est une des rares cuvres que l'on peut raconter. De ce fait, cette pièce $a$, dans la production de cet auteur, une valeur particulière, une pièce charnière, en quelque sorte. Tout ce qu'il a écrit depuis le début s'y retrouve : cette simplification, cette manière de jouer avec les mots, cette manière de parler pour ne rien dire, qui est une façon dramatique de parler théâtralement, d'occuper l'espace avec des mots sans qu'il y ait d'action, cette espèce d'immobilité ou il y a un mouvement de la conscience et de l'inconscient, mais où il n'y a pas d'actions ou de faits concrets Dans $L e$ Roi se Meurt, en entrant en scène, le personnage dit "Je vais mourir", et il finit par mourir, voilà toute l'histoire. Mais cette longue agonie dure trois heures. Le thème de la mort, la présence, l'obssession, la crainte de la mort sont présentes d'une manière dramatique et d'une manière dérisoire jusqu'au bout parceque la mort finit par vaincre. Macbett est une œuvre intéressante car elle emprunte à Shakespeare les personnages, - qu'Ionesco déforme - et l'anecdote.

JMD : Le texte de Shakespeare est parfois cité littéralement, notamment à la fin, le discours de Malcoll est celui que l'on trouve dans la scène où Malcolm met MacDuff à l'épreuve.

JL : Mais là, cette scène est complètement déplacée. 
JMD : Arraché au contexte, si bien que l'épisode prend un tout autre sens et devient une glorification éhontée de la tyrannie. Ionesco a tout de même une fascination pour Shakespeare. Le Roi Lear a un rapport avec Le Roi se Meurt, ou tout au moins une certaine lecture du Roi Lear, comme Fin de Partie.

$\mathrm{JL}$ : Il y a aussi la mauvaise foi de Ionesco qui se mélange. Cette partie qu'il emprunte directement à Shakespeare pour finir la pièce est un discours qu'il écrit entièrement au négatif. On a fait la méme confusion du bien et du mal dans Macbeth, mais c'est le triomphe du mal, de la sorcellerie, du diable. C'est la création d'un immense désordre, mais à la fin, comme dans presque toutes les pièces de Shakespeare, l'ordre est à nouveau rétabli; le fils de Duncan arrive et promet une société meilleure, ou tout au moins une société dans le respect de tout ce qui a été transgressé et dont l'ordre va être rétabli. Ici, c'est à l'envers, tous les discours du personnage sont rendus d'une manière négative, négativiste. Ionesco pense qu'il est quelque part le porte-parole de Shakespeare, et que ce message négativiste est le message que Shakespeare pensait, mais que pour des raisons politiques il ne pouvait pas écrire.

JMD : Ne pensez-vous pas qu'Ionesco a été influencé par l'ouvrage de Jan Kott?

JL : Comme la pièce a été écrite en 1972 , et peut-être conçue avant, il est évident que comme tout le monde il a été influencé par Kott. Mais en rendant le négativisme total et absolu pire que dans Shakespeare, et en enfonçant le clou à ce point, Ionesco est Ionesco $100 \%$. C'est l'anarchiste négativiste qui ne croit pas du tout aux valeurs de l'espèce et qui ne croit pas surtout aux valeurs politiques. Au moment où il a écrit Macbett, on est en pleine époque positiviste, optimiste, avec une Brecht triomphant sur tout le théâtre en Europe. Puis vient cette pièce qui nie toute qualité à l'homme politique, la pièce ne pouvait pas avoir un écho positif. C'est curieux comme vingt ans de différence ont fait un certain travail, on a dépassionné le débat, on n'a pas besoin aujourd'hui d'être optimiste à outrance.

JMD : De telle sorte que l'on a l'impression que la pièce est plus d'actualité en 1992 qu'en 1972, surtout après l'épisode Caucescu, auquel on pense constamment.

$\mathrm{JL}$ : Evidemment Ionesco a dû penser à son pays. 
JMD : C'est comme une prémonition aussi du phénomène Caucescu et de la caricature qui était donnée du pouvoir.

JL : Peut-être a-t-on aussi vécu beaucoup d'événements qui sont parallèles et qui ont contribué à ce que la lecture ne se fasse pas simplement à travers l'auteur ou à travers la mise en scène, mais aussi à travers le public. J'ai été frappé par certaines scènes relativement drôles, mais on n'attend pas que le public puisse rire. Par exemple, un soldat en amène un autre, un troisième personnage l'interpelle en lui disant "il est blessé ?" -"non, il est mort". Et la salle rit, et la deuxième fois, on rit davantage. C'est-à-dire que, à mon avis, il y a une saturation de la mort à travers l'information depuis la guerre du Golfe et peut-ètre d'autres guerres, si bien que la mort a perdu un peu de son prestige. Même si la situation est dérisoire, on ne peut pas prévoir une réaction de ce type, tout du moins à ce moment-lâ. L'actualité est parfois un élément démagogique au théâtre, mais je crois qu'il y a comme une maturation, un mûrissement, si bien que l'on peut regarder certain événements avec plus d'objectivité. Et c'est justement grâce à cette objectivité-là que l'on peut rire du chaos, du désordre qui est raconté dans la pièce d'Ionesco.

JMD : Des participants ont-ils vu le spectacle?

Guy Boquet : Je reviendrai sur quelque chose du début qui replace bien la scène dans le cadre roumain : deux généraux de Malcoll portent un short noir ... On voit ensuite une scène à peu près identique entre Macbett et des personnages qui portent le même costume, mais rouge. Je suppose que vous avez voulu par là montrer une référence sous-jacente dans l'œuvre d'Ionesco : le passé nazi de la Roumanie et le communisme qui se suivent.

$\mathrm{JL}$ : Je n'ai pas tellement pensé à cela. Voyez comme c'est une œuvre ouverte, on voit bien que le théâtre laisse la place aux interprétations. Votre interprétation me convient assez bien. J'avais tout simplement pensé au footing, j'avais aussi pensé au thème du dualisme pirandellien, ses personnages sont toujours doubles: Macbett et Banco tiennent le même discours, ils prononcent exactement les mêmes mots. C'est une des choses qui m'a le plus frappé à la première lecture de cette pièce que j'ai eu l'occasion de redécouvrir au cours d'un petit travail que j'avais fait pour la re-publication des œuvres complètes d'Ionesco dans la Pléiade. C'était une pièce que l'on avait un peu abandonné, oublié. Ce sont 
deux généraux un peu nalfs, un peu simplets : Banco est plus prêt à une obéissance consciente et plus sérieuse, Macbett est un personnage un peu plus effacé, avec moins de trace de rigueur, à la limite du "pauvre type".

JMD : Que Michel Aumont interprète superbement.

$\mathrm{JL}$ : Ce sont des porte-paroles, des figures de théâtre, des caricatures. Ils deviennent plus intéressants au fur et à mesure qu'ils passent un certain nombre d'épreuves. Ils sont très linéaires au départ avec une grande fidélité pour Duncan et ils basculent assez vite dans la trahison. Ils pensent qu'une fois sous le charme du surnaturel, ils commencent à avoir une certaine imagination, une certaine réflexion. Ils commencent aussi à devenir complexes. C'est ce qui est intéressant dans la démarche d'Ionesco : des figures presque caricaturales, navrantes de simplicité, s'enrichissent des épreuves qu'ils doivent affronter. C'est une manière de regarder l'œuvre de Shakespeare, de la critiquer, et aussi de l'aimer parce qu'il a mis en scène aussi les sorcières, qui ne sont pas trois d'ailleurs, mais deux. Ce dualisme qui existe dans les autres personnages dépasse peut-être le sens de l'économie, car Ionesco est un auteur économe dans l'invention de ses personnages; d'habitude, il n'y a pas prolifération de personnages dans ses pièces. Mais ici, il y a cette espèce de partage de la personnalité, un personnage est toujours le reflet de l'autre, l'écho de l'autre. Ce dualisme un peu pirandellien, se continue jusqu'au bout. C'est une idée dramaturgique très intéressante qui est une manière d'aborder l'identité.

Guy Boquet: A propos des sorcières, un personnage important disparaît, il s'agit de Iady Macbett remplacée par une sorcière qui va trahir Duncan. Les deux sorcières se livrent ensuite à une sorte de strip-tease ; l'une va séduire Macbett on comprend ensuite qu'elle va se faire passer pour la femme de Duncan pour redevenir Lady Macbett. Ce rôle comporte en fait deux rôles: celui de Lady Duncan puis de la sorcière, remarquablement tenu par Isabelle Cayat.

JMD : Qui avait créé cette pièce?

$\mathrm{JL}$ : C'était dans le petit théâtre de l'Alliance Française en 1972, dans une mise en scène de Jacques Mauclair.

Gisèle Venet : Pour reprendre la différence entre la création et ce que vous avez fait. Mauclair avait conservé scéniquement 
cette symétrie : les personnages apparaissaient symétriques, récitaient symétrique et l'abstraction était plus grande. J'oserai dire que vous avez donné une version plus brechtienne.

JMD : En quel sens?

GV : Chez Mauclair, il y avait plus d'ironie du verbe, au fond il n'y avait presque pas de jeu, c'était plus statique, tandis que vous, vous avez un parti-pris de mise en scène, la violence est exacerbée, il y a une lecture d'Ionesco en plus.

Guy Boquet : En tant que modeste praticien du théâtre scolaire et universitaire, je pense qu'on a une donnée, c'est la convention du plateau. Si Jacques Mauclair avait eu un théâtre aussi grand que celui de la Colline, il aurait fait quelque chose d'autre. Vous auriez été obligé de faire une autre mise en scène si vous aviez eu le petit théâtre de l'Alliance Française.

$\mathrm{JL}$ : C'est vrai que la mise en scène et l'architecture sont des choses intimenent liées. Cette pièce a été faite pour le grand plateau de la Colline qui fait deux fois sinon trois le plateau de l'Alliance Française d'après mes souvenirs. Ce qu'on avait vu au Théâtre de la Colline et que l'on voit maintenant à Lyon où la pièce continue à se jouer en tournée, c'est un labyrinthe, c'est une idée complètement différente dans le sens où les personnages sont inscrits dans un mouvements labyrinthique, où le temps est quelque part aboli. Même si les costumes sont relativement modernes, ils donnent une fonction aux personnages : les militaires sont tous ceux qui décident et qui finissent par avoir le pouvoir. Le fils de Duncan est aussi un militaire bien qu'il soit habillé en "marine américain", comme on a pu les voir pendant la guerre. Ces anachronismcs sont présents dans la pièce d'Ionesco, ils sont aussi présents dans ma mise en scène, mais ce sont des anachronismes plus proches de nous, plus modernes: les armes, les uniformes. Il n'y a pas ce saut immense entre la Renaissance et aujourd'hui. Il y a aussi des choses qui sont propres à un langage, à une écriture de mise en scène. Les sorcières, qui font partie du surnatuel, utilisent des éléments qui sont peut-être brechtiens, mais qui en tout cas sont des éléments modernes: ce ne sont pas des socles, c'est ce qui est utilisé couramment dans l'industrie pour faire déplacer les peintres, par exemple, en avant, en arrière, en haut et en bas. Ce ne sont pas des socles classiques de thêâtre, dirigés par deux personnes, voire par trois. Au départ donc, ce sont 
dcs éléments de travail. Ainsi, ils donnent la liberté aux interprètes, en l'occurrence aux deux sorcières, d'auto-gérer leur travail. Bien sûr tout cela a été travaillé pendant longtemps, ce qui permet parfois aux interprètes d'utiliser cet élément de travail d'une manière non-orthodoxe.

GV : Les sorcières font penser à Mary Poppins dans les déplacements, dans les costumes. Pourrions-nous parler de la manière dont vous privilégiez l'entrainement physique à outrance : les personnages disent leur texte en faisant des pompes. La dégénérescence du pouvoir prend le chemin d'une dégénérescence de l'entraînement physique. Dans le cas de Malcoll, cela m'a semblé arriver à un point qui était à la limite de la crédibilité : Malcoll débitant son texte en tournoyant comme un possédé sur son siège était très excessive. Je me suis demandé si vous n'aviez pas été victime de votre parti-pris initial de mise en scène.

JL Je crois que vous avez raison. Le rôle a beaucoup évolué. On ne fait pas une mise en scène dans l'absolu car on doit penser par rapport à un espace, et on ne peut pas emprisonner le personnage dans une première vision. Au départ, dans mon esprit, ce devait être un enfant, donc il était habillé comme un enfant. Mais il était extrêmement drôle comme une blague peut être drôle, donc cela ne m'a pas plu. Il a changé et il est devenu un marin comme on habille les enfants en marin, mais propre, il n'était pas mieux, il n'était pas assez dangereux. C'est très difficile parfois de combattre un costume: on ne peut pas abonder dans le sens du costume, cela ferait pléonasme. Il devait garder un côté très jeune, pas enfant, et son costume est un costume de marin d'après-guerre mais il a été coupé : le pantalon ne va pas jusqu 'aux chaussures, donc il y a déjà un côté enfantin, ou jeune homme. Il ne vient pas en bateau, mais sur sa moto américaine. Le fait de fonctionner un peu comme un schizophrène me plaisait beaucoup : il ne devait pas être un militaire commme les autres, mais un militaire encore plus fou que tous les autres, plus irrationnel, surtout qu'il était le fruit d'un texte emprunté à Shakespeare et déformé, transformé par Ionesco. Un discours tout à fait rassurant dans Shakespeare devient ici inquiétant et menaçant, donc totalement schizophrène. Cet acteur a une très grande mobilité, une très grande force, un très grand entraînement physique, c'est presque un athlète : il se laissait tomber il bougeait avec une très grande violence comme un personnage en caoutchouc. Je le trouvais fascinant parcequ'on ne pouvait 
pas le cerner d'un point de vue psychologique, commme un fou délirant noyé dès le départ dans le pouvoir.

Margaret Jones-Davies : En voyant cette pièce j'ai été fascinée par ce thème de l'infantilisme. La violence prend sa source dans cette régression infantile. J'ai aimé ce personnage de Duncan qui regarde les exécutions capitales en suçant son pouce en position fœtale dans un fauteuil. En relisant le texte, j'ai été frappée par le fait que Malcoll n'est pas le fils de Duncan, mais le fils adoptif, ses vrais parents sont Banco et une gazelle ; cette gazelle aurait été métamorphosée en femme par une sorcière. Pourquoi Ionesco passe-t-il du fils au fils adoptif, en avez-vous une interprétation?

JL : Il doit être le successeur de Banco pour établir la dynastie de Banco, donc, c'est un raccourci.

M.J-D. : Le fils du roi usurpe l'autre dynastie. Dans le texte, tout de suite après. C'est le thème œdipien qui ressort très clairement.

JMD : La pièce se termine par un chasseur de papillons. Comment peut-on interpréter cela?

JL : Souvent, dans les pièces d'Ionesco, lorsqu'on déverse beaucoup de méchanceté ou de désordre, quelqu'un passe en maniant un balai. Ce n'est pas dans L'Impromptu de Versailles, mais dans L'Impromptu de l'Alma. Des intellectuels brechtiens discutent sur le théâtre, et la femme de ménage passe avec un balai et balaye tout. Il y a un peu cette allusion dans l'idée du chasseur de papillons. La différence, c'est que le chasseur de papillons nous fait rêver davantage, c'est plus poétique, c'est quelqu'un qui va à la recherche de quelque chose de rare, peut-être poursuit-il la vérité, l'idée me semblait très belle et je l'ai gardée avec plaisir.

JMD : Mon interprétation était sans doute trop simpliste. Le pouvoir est corrompu, on nous en montre la comédie grinçante, mais il y a peut-être l'espoir ténu de la poésie, l'espoir est à chercher, il est en dehors du politique.

JL: Du fait même que le poète est vivant, je pensais qu'il y a toujours de l'espoir. Sur le plan de l'interprétation scénique, ce papillon est terre-à-terre, mais évoque peut-être quelque chose d'autre, la vérité comme vous le suggérez, qui nous échappe. 
GV : Je voudrais que vous nous parliez de l'intrusion du quotidien, la mère qui abreuve ses enfants, ce qui m'a semblé être une caricature.

$\mathrm{JL}:$ Je ne sais pas si c'est une caricature. Peut-être a-t-il pensé à La Mère Courage de Brecht. Un limonadier sort vendre sa limonade en pleine guerre. Il fait son petit commerce, et rencontre un soldat terriblement exigeant qui finit par le tuer. Voilà les coups de théâtre inattendus d'Tonesco qui font parfois rêver. Cet auteur a une telle imagination, dans le simple échange de trois répliques, il peut revenir à des situations complètemcnt quotidiennes, banales, confrontées à quelque chose de tragique qu'on ne voit pas, la guerre. Il introduit un autre personnage, ce soldat anonyme, qui se sert du limonadier et qui finit, pour rien, juste pour la violence pure, par le tuer. Une scène de deux minutes vous laisse perplexe avec cette invention dramatique.

JMD : N'avez-vous pas le sentiment que Ionesco restera plus pour sa poétique comique, le chasseur de papillons, la gazelle, etc., que par son discours sur le politique qui est d'un niveau réducteur, et un peu sommaire.

$\mathrm{JL}:$ Je trouve que la pièce est très intéressante parce qu'Ionesco s'approprie un thème, une légende, presque.

JMD : Il est complètement nihiliste, si l'on compare avec la ré-écritue que Bond fait du Roi Lear, pièce très noire et désespérée mais où malgré tout il y a une tentative de construction, d'un renouveau possible, par une réforme morale chez l'homme. Pour Ionesco, il n'y a pas d'espoir. Les hommes politiques sont une série de pantins caricaturaux.

JL : Bond est lui aussi très dur en politique. Il croyait, dans les années soixante-dix, à la construction d'une nouvelle société à travers le marxisme.

JMD : Bond est plus un moraliste qu'un marxiste.

Marie-Thérèse Jones-Davies : Ionesco fait montre d'humour quand il prend la direction de la cour.

JL : Oui, n'importe qui peut être roi, y compris l'auteur. C'est une sorte de pirouette à laquelle évidemment Ionesco se prête avec plaisir.

Ce n'est peut-être pas l'œuvre capitale d'Ionesco, mais c'est 
une œuvre qui concentre un peu toutes les recherches de l'auteur depuis l'exercice de style jusqu'à l'impresion d'une certaine pensée. C'est une clarification de sa position, sinon politique, du moins nihiliste par rapport à la mort. On le savait angoissé sur le problème de la mort, toute son œuvre en témoigne. C'est quelqu'un qui, même avec humour, avec ironie grinçante, est d'un scepticisme total et absolu. Ce qui n'est peut-être pas nouveau, ni dans le théâtre ni dans la nature, c'est que cette vision complètement noire, négativiste est chargée d'humour. C'est aussi une manière de secouer, d'interpréter la conscience des gens. C'est une manière aussi de reposer la question du pouvoir, même si son point de vue est effectivement assez réducteur.

J'ai monté quelques pièces de Shakespeare, dont $\mathrm{La}$ Tempête, Le Songe d'une Nuit d'été, Le Conte d'hiver, qui a été à Avignon, et il y a très longtemps, Beaucoup de bruit pour rien. Depuis, je m'interdis Shakespeare, parce que tout simplement, avec l'aventure de La Colline, je me trouve sur une autre planète, dans un théâtre de découverte, pari difficile et en tous cas très intéressant. Ce Théâtre prouve qu'à l'intérieur d'une grande ville comme Paris, dans cette géographie nationale et théâtrale, il est possible d'imaginer un théâtre de création, un théâtre professionnel qui n'est pas un théâtre de recherche ni expérimental ni de laboratoire, mais un théâtre qui essaie de transcrire la réalité artistique et socio-politique de notre temps à travers des auteurs français ou étrangers que nous considérons comme importants. Ce théâtre gagne de plus en plus de spectateurs. Cela me console de ne pas pouvoir monter de pièce de Shakespeare que j'aime beaucoup.

JMD : Il nous reste à vous remercier très chaleureusement d'être venu nous parler aussi spontanément et si généreusement de votre travail sur Ionesco. A-t-il vu le spectacle?

JL : Oui, le jour de la première, et le 31 décembre, la dernière représentation à Paris.

Marie-Thérèse Jones-Davies : C'était très impressionnant. Il a eu une très longue ovation. 